RESEARCH

\title{
Androgens by immunoassay and mass spectrometry in children with $46, X Y$ disorder of sex development
}

\author{
Letícia Ribeiro Oliveira ${ }^{1,2}$, Carlos Alberto Longui ${ }^{3}$, Guilherme Guaragna-Filho $0^{1,4}$, José Luiz Costa ${ }^{5,6}$, Rafael Lanaro ${ }^{6}$, \\ David Antônio Silva ${ }^{7}$, Maria Izabel Chiamolera ${ }^{8}$, Maricilda Palandi de Mello ${ }^{1,9}$, André Moreno Morcillo ${ }^{10}$, \\ Andrea Trevas Maciel-Guerra ${ }^{1,11}$ and Gil Guerra-Junior ${ }^{1,10}$

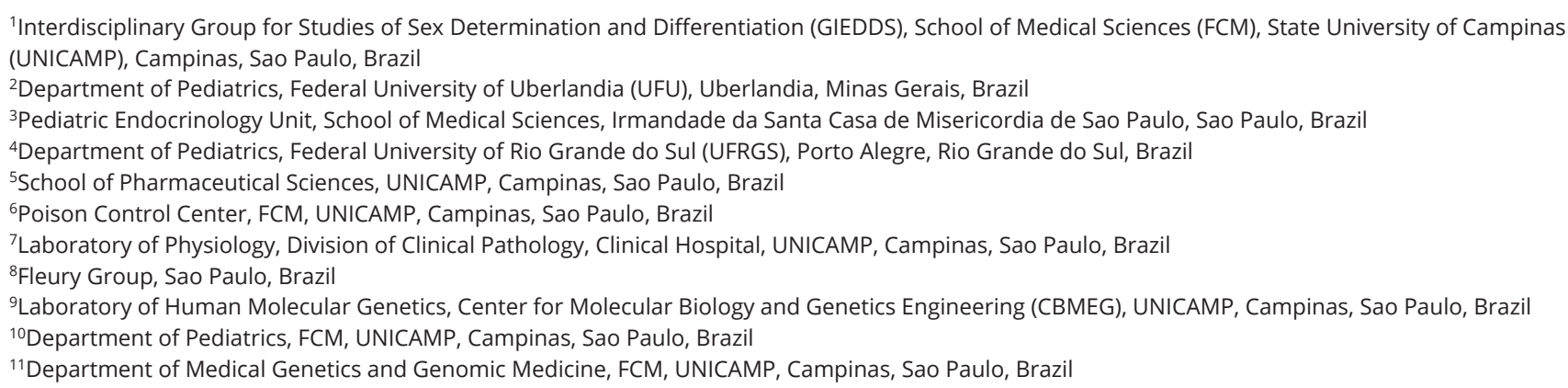

Correspondence should be addressed to G Guerra-Junior: gilguer@unicamp.br

\begin{abstract}
Objective: Steroid measurement is a challenge in pediatric endocrinology. Currently, liquid chromatography with tandem mass spectrometry (LC-MS/MS) is considered a gold standard for this purpose. The aim of this study was to compare both LC-MS/MS and immunoassay (IA) for androgens before and after human recombinant chorionic gonadotropin (rhCG) stimulus in children with 46,XY disorders of sex development (DSD). Methods: Nineteen patients with 46,XY DSD were evaluated; all of them were prepubertal and non-gonadectomized. Testosterone, dihydrotestosterone (DHT), DHEA and androstenedione were measured by IA and LC-MS/MS before and 7 days after rhCG injection. The correlation between IA and LC-MS/MS was analyzed by the intraclass correlation coefficient (ICC) and Spearman's rank correlation coefficient (SCC). For concordance analysis the Passing and Bablok (PB) regression and the Bland and Altman (BA) method were used.

Results: Testosterone showed excellent correlation (ICC $=0.960$ and SCC $=0.964)$; $\mathrm{DHT}$ showed insignificant and moderate correlations as indicated by ICC (0.222) and SCC (0.631), respectively; DHEA showed moderate correlation $(\mathrm{ICC}=0.585$ and $\mathrm{SCC}=0.716)$; and androstenedione had poor and moderate correlations in ICC (0.363) and SCC (0.735), respectively. Using the PB method, all hormones showed a linear correlation, but proportional and systematic concordance errors were detected for androstenedione, systematic errors for testosterone and no errors for DHEA and DHT. By the BA method, there was a trend of IA to overestimate testosterone and androstenedione and underestimate DHEA and DHT when compared to LC-MS/MS.

Conclusion: Traditional IA should be replaced by LC-MS/MS for the androgens measurement in prepubertal children whenever is possible.
\end{abstract}

\author{
Key Words \\ - testosterone \\ - testicles \\ - androstenedione \\ - androgen insensitivity \\ syndrome \\ - $5 \alpha$-reductase
}

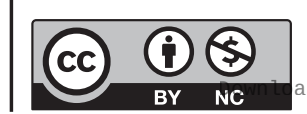

This work is licensed under a Creative Commons Attribution-NonCommercial 4.0 International License. ded from Bioscientifica.com at 04/26/2023 12:08:09PM
Endocrine Connections (2020) 9, 1085-1094 


\section{Introduction}

The determination of steroid hormone concentration, which is part of the initial approach in the diagnosis of disorders of sex development (DSD), is one of the challenges in pediatric endocrinology $(1,2,3)$. Based on the principle of antigen-antibody complex formation, immunoassays (IA) are widely used in clinical practice due to its low cost, easy technical application and reproducibility $(2,3,4)$. However, IA for the measurement of analytes such as the steroids that presents low antigenic activity do not offer proper specificity $(1,3)$.

Conventional IA currently available are not accurate for the evaluation of substances with low molecular weight and immunogenicity due to cross-reaction frequently occurring with precursors, metabolites and components of the assay matrix. In addition, samples with higher volume are necessary because only one steroid is dosed per analysis and steroid hormone concentrations are generally low $(1,5)$.

The use of liquid or gas chromatography coupled to mass spectrometry (MS) is frequently used for steroid analyses $(1,3,4)$. These techniques are more specific and more accurate than IA (6), however, they are still highcost methods and are not available as routine tests in most laboratories $(3,4)$.

More recently, liquid chromatography coupled with mass spectrometry in tandem (LC-MS/MS) has replaced conventional methods of steroid hormone analysis, enabling the use of small volume samples, improving specificity and reducing the time of analyses $(3,7,8)$. Such method is recognized as the gold standard for steroid dosage when properly used and under highquality technical control. Despite all advantages, LC-MS/MS is still limited since it is not a technology that offers complete automation $(1,7,8)$.

The use of LC-MS/MS in research laboratories has expanded $(3,4)$, because it has proved useful in improving the accuracy of steroid measurements, especially in low concentration samples, as observed in children with DSD (2). One of the most important challenge is the measurement of testosterone concentration $(9,10)$, especially in the investigation of 46,XY DSD cases.

DSD are congenital conditions in which chromosomal, gonadal or anatomical sex is atypical. Patients with male karyotype and DSD (46,XY DSD) may present female or ambiguous external genitalia, late or atypical puberty, or only infertility $(11,12)$.

The initial investigation of patients with ambiguous genitalia, 46,XY karyotype and testes consists on investigating whether there is adequate production of testosterone, which depends on quantity and quality of the testicular tissue $(11,12)$. Most testicular 46,XY DSD cases present normal testosterone production and include complete androgen insensitivity syndrome (CAIS) (OMIM \#300068) or partial androgen insensitivity syndrome (PAIS) (OMIM \#312300) caused by mutations in the $A R$ (OMIM *313700) gene, and $5 \alpha$-reductase type 2 deficiency (5AR2D) (OMIM \#264600) caused by mutations in SRD5A2 gene (OMIM *607306). The idiopathic cases correspond $30 \%$ to $50 \%$ of testicular $46, \mathrm{XY}$ DSD with normal testosterone secretion $(13,14)$.

The human chorionic gonadotropin (hCG) stimulation test is performed to evaluate gonadal steroidogenic secretion $(11,12,15)$. The hCG purified from pregnant women's urine (uhCG) has been used for this purpose, based on well-established protocols $(15,16)$. A recent alternative for this test has been the use of hCG produced by recombinant DNA techniques (rhCG) (15, $17,18)$.

Some studies comparing limits, advantages and disadvantages of steroid levels using IA and LC-MS/MS methods are available in the literature $(1,4,19,20)$ but none of them have compared the performance of such methods to assess androgen secretion under hCG stimulus conditions in children.

Therefore, the aim of this study was to compare the levels of androgens assessed by conventional IA and by LC-MS/MS before and after the stimulation with rhCG in prepubertal children with $46, \mathrm{XY}$ DSD diagnosis.

\section{Materials and methods}

\section{Clinical data}

Our sample comprised 19 prepubertal children with testicular 46,XY DSD, not gonadectomized, and proven normal testosterone secretion evaluated by conventional IA after uhCG stimulation test in childhood (total testosterone higher than $1.5 \mathrm{ng} / \mathrm{mL}(15,21))$ or hormonal assessment done during mini-puberty (total testosterone higher than $1.5 \mathrm{ng} / \mathrm{mL}$ ). The sample included 5 cases of PAIS, 4 cases of 5AR2D and 10 cases of idiopathic $46, \mathrm{XY}$ DSD. These idiopathic cases had normal testosterone secretion and molecular study with no pathogenic variants for AR, SRD5A2 and NR5A1 (OMIM *184757).

Patients in this study were selected from a sample of 408 patients, described in 2016 (14) with DSD diagnosis at the Interdisciplinary Study Group for Sex Determination

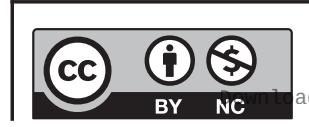


and Differentiation (GIEDDS) between 1989 and 2016, among which, 189 presented 46,XY karyotype and bilateral testes, and 107 presented normal testosterone secretion. Among these 107 cases, 10 were diagnosed with PAIS (five prepubertal, four in puberty and one gonadectomized); 20 were diagnosed with 5AR2D (5 prepubertal, 8 in puberty and 7 gonadectomized) and 77 with idiopathic 46,XY DSD (15 prepubertal, 61 in puberty and one gonadectomized). Thus, 25 patients within this sample fit the inclusion criteria for the study and 19 are still followed up in service, and accepted to participate in the study.

All patients were evaluated at the GIEDDS Outpatient Clinic of Clinical Hospital of the State University of Campinas (UNICAMP) and they were accompanied by a responsible adult who signed a free and informed consent form according to the Declaration of Helsinki. This study was approved by Research Ethics Committee of UNICAMP (Process number: 48063115.4.0000.5404).

The study was prospective and cross-sectional, and the children were evaluated in two time-points: at the first clinical evaluation, when a blood sample was taken for basal hormonal analysis and rhCG application; and, at 7 days after the first evaluation, for obtaining a new blood sample for hormonal analysis and clinical evaluation. Immediately after the basal sampling, one rhCG (Ovidrel ${ }^{\circledR}$ - Merck Serono) ampoule of $250 \mu \mathrm{g}$ (6500 IU) was applied subcutaneously by the main investigator of the study.

\section{IA data}

Hormones assessed at the two moments of the study were total testosterone, dihydrotestosterone (DHT), dehydroepiandrosterone (DHEA) and androstenedione. Each one was evaluated by IA and LC-MS/MS at both time of the study.

Concerning IA methods, total testosterone was assessed by electrochemiluminescence (ECLIA) (Roche, Germany, Cobas E, 05200067190, detection limit: 0.03-15 ng/mL); DHT by enzyme immunoassay (ELISA) (DBC - Diagnostic Biochem Canada Inc, CAN-DHT-280, sensitivity of $6 \mathrm{pg} / \mathrm{mL}$ ); DHEA by ELISA (DBC - Diagnostic Biochem Canada Inc., CAN-DH-490, sensitivity of 0.15 $\mathrm{ng} / \mathrm{mL}$ ) and, androstenedione by ELISA (DBC - Diagnostic Biochem Canada Inc, CAN-AD-208, sensitivity of $0.04 \mathrm{ng} / \mathrm{mL}$ ).

The type of antibody used in each immunoassay was: biotinylated MAB (sheep) $40 \mathrm{ng} / \mathrm{mL}, 2$-bromoestradiol release reagent $50 \mathrm{nmol} / \mathrm{L}$, $\mathrm{pH} 6.0$ preservative for testosterone electrochemiluminescence assay, polyclonal
anti-DHEA (rabbit) antibody in a 96-well microplate for DHEA enzymatic assay, polyclonal (rabbit) antibody in a 96-well microplate containing a desiccant was used for androstenedione and DHT enzymatic assays.

\section{LC-MS/MS data}

\section{Standards and chemicals}

Reference materials of testosterone (1 $\mathrm{mg} / \mathrm{mL}$ ), dihydrotestosterone (DHT, $1 \mathrm{mg} / \mathrm{mL}$ ) were purchased from LGC Standards (Luckenwalde, Germany). Reference materials of DHEA $(1 \mathrm{mg} / \mathrm{mL})$, androstenedione $(1 \mathrm{mg} / \mathrm{mL})$, testosterone-D3 $(0.1 \mathrm{mg} / \mathrm{mL})$ and DHEA-D5 $(0.1 \mathrm{mg} / \mathrm{mL})$ were purchased from Cerilliant (Round Rock, TX, USA). Charcoal-stripped fetal bovine serum (hormone free), acetonitrile e methyl-terc-butyl ether (HPLC grade) were purchased from Sigma-Aldrich. Ultrapure deionized water was supplied by a Milli-Q ${ }^{\mathrm{TM}}$ RG unit from Millipore.

DHT concentrations were quantified by itself through a fully validated laboratory-developed test (property of Fleury Lab), using LC-MS/MS (Waters Corporation) and reversed-phase column, serum samples $(250 \mu \mathrm{L})$ were submitted to isotopic dilution, followed by analyte enrichment. Sample separation was done via liquid chromatography and the detection via mass spectrometry in tandem. Monitoring ion precursors of $\mathrm{m} / \mathrm{z} 184$ did the presence of phospholipids. The liquid-liquid extraction process was standardized by the maximum removal of these interfering and the DHT coelution with phospholipids and other low molecular weight compounds ( $m / z$ 50-500) was not detected. Over 50 sequenced samples were analyzed. Also, some specific DHT endogenously found isomers were tested (androsterone and etiocholanolone), besides testosterone, which is one of the main endogenously interfering elements of this analyte (present in much greater concentrations than DHT in serum). Considering the endogenous concentrations of these steroids, interference was not observed. To DHT analysis, the quantification limit was of $5 \mathrm{ng} / \mathrm{dL}$ and the linearity was of $2800 \mathrm{pg} / \mathrm{mL}$ (280 ng/dL). Total imprecision for low and high concentration was lower than $6.5 \%$.

\section{Standard solutions and calibrators}

The stock solution was prepared by dilution of reference materials in methanol. Calibrators and quality control samples were prepared by spiking charcoal-stripped fetal bovine serum in order to achieve concentrations from 0.05 to $10 \mathrm{ng} / \mathrm{mL}$ to all hormones (except to DHEA, from 0.25 to $10 \mathrm{ng} / \mathrm{mL})$. Dextran treated charcoal is used to 
selectively remove hormones without nonspecific loss of other serum components.

\section{Sample preparation}

Serum samples $(500 \mu \mathrm{L})$ were transferred to polypropylene tubes, followed by $25 \mu \mathrm{L}$ of internal standard solution (testosterone-D3, $10 \mathrm{ng} / \mathrm{mL}$ in methanol) and $4 \mathrm{~mL}$ of methyl-terc-butyl ether. The tubes were capped, homogenized by inversion 10 times and vortexed by 1 min and then centrifuged at $4000 \mathrm{rpm} / 5 \mathrm{~min}$. The organic phase was transferred to conical glass tube and evaporated to dryness under nitrogen stream. The residue was reconstituted with $150 \mu \mathrm{L}$ of acetonitrile:ultrapure water and $30 \mu \mathrm{L}$ was injected into LC-MS/MS system.

\section{Instrumentation}

The analyses were performed on a 1260 Infinity liquid chromatography system (Agilent Technologies) coupled to a 5500-QTRAP ${ }^{\circledR}$ hybrid triple quadrupole mass spectrometer (ABSciex, Concord, ON, Canada).

The chromatographic separation was performed with a Biphenyl column (Kinetex 2,6u biphenyl 100A, $100 \times 2.1 \mathrm{~mm}$, Phenomenex, Torrance, CA, USA), maintained at $40^{\circ} \mathrm{C}$. The mobile phase consisted of ultrapure water (A) and acetonitrile (B), both containing formic acid $(0.1 \%, \mathrm{v} / \mathrm{v})$. The gradient elution was programmed as follows: $10 \% \mathrm{~B}$, followed by a linear change to $95 \%$ B over 2 min, held at $95 \%$ B for $4.5 \mathrm{~min}$ and returned to initial conditions over $0.2 \mathrm{~min}$, with extra $6 \mathrm{~min}$ of equilibration time. The mobile phase flow rate was $0.3 \mathrm{~mL} / \mathrm{min}$.

The 5500-QTRAP ${ }^{\circledR}$ mass spectrometer was equipped with a TurboIonSpray ${ }^{\mathrm{TM}}$ interface using electrospray ionization in the positive ionization mode. Nitrogen was used as curtain, collision and nebulizer gas. The source parameters were: ion source temperature, $600^{\circ} \mathrm{C}$; ion spray voltage, $5.5 \mathrm{kV}$; entrance potential (EP), $10 \mathrm{~V}$; nebulizer gas (GS1) pressure, 70 psi; auxiliary gas (GS2) pressure, $40 \mathrm{psi}$; and curtain gas pressure, 10 psi. The analyses were performed in multiple reaction monitoring (MRM) mode. For each compound, two MRM transitions were chosen for quantification and confirmation, and optimized by constant infusion of working solutions of each analyte (20 ng/mL in water/methanol, 1:1, v/v). Analyst 1.6.2 software was used for data collection and MultiQuant 3.0.1 for data processing. Before sample analysis, the method was validated using charcoalstripped fetal bovine serum fortified with reference materials. Parameters evaluated during method validation were sensitivity (limit of detection and quantitation) and linearity, specificity, accuracy and precision, recovery and carryover, according to international guidelines (22).

Data about MRM transitions and retention times for evaluated hormones are shown in Table 1.

\section{Statistical analysis}

The distribution of each steroid by IA and LC-MS/MS was verified before and after rhCG stimulus using Kolmogorov-Sminorv test. Since all distributions were not normal, nonparametric Kruskal-Wallis test was used to compare the steroid differences among the three groups in both trials and on both moments also paired Wilcoxon test to verify the differences on each steroid on each trial between the two periods. All cases on both collection times were grouped to analyze the correlation and concordance between IA and LC-MS/MS.

For the correlation analysis the intraclass coefficient (ICC) and the Spearman's correlation coefficient (SCC) were used. For the concordance analysis the Passing and Bablok (PB) regression (23) and Bland and Altman (BA) plot (24) were used. Comparisons were made assuming LC-MS/MS assay as the reference method. For ICC the following analysis criteria were used (25):

Table 1 MRM optimized transitions and retention time for analysis of testosterone, DHEA, testosterone-d3 (IS1), and androstenedione by liquid chromatography-tandem mass spectrometry (LC-MS/MS).

\begin{tabular}{|c|c|c|}
\hline Compound & Retention time (min) & \\
\hline Testosterone & 6.57 & \\
\hline DHEA & 6.58 & \\
\hline Androstenedione & 6.84 & \\
\hline Testosterone-d3 (IS1) & 6.58 & \\
\hline \multicolumn{3}{|c|}{ CXP, cell exit potential; DP, declustering potential; MRM, multiple reaction $\mathrm{m}$} \\
\hline $\begin{array}{l}\text { https://ec.bioscientifica.com } \\
\text { https://doi.org/10.1530/EC-20-0454 }\end{array}$ & $\begin{array}{r}\text { (C) } 2020 \mathrm{~T} \\
\text { Published by Biosci }\end{array}$ & \\
\hline
\end{tabular}

\begin{aligned} \multicolumn{1}{c}{ MRM } \\ \hline $288.8 \rightarrow 97.1 \\ 288.8 \rightarrow 109.1 \\ 288.8 \rightarrow 91.0 \\ 288.8 \rightarrow 253.1 \\ 286.8 \rightarrow 97.1 \\ 286.8 \rightarrow 109.1 \\ 291.8 \rightarrow 97.1 \\ 291.8 \rightarrow 109.1\end{aligned}$

\begin{tabular}{c}
\hline Collision energy $(\mathrm{eV})$ \\
\hline 27 \\
29 \\
57 \\
13 \\
25 \\
27 \\
27 \\
29
\end{tabular}

\begin{tabular}{ccc}
\hline $\mathbf{D P}(\mathrm{V})$ & & $\mathbf{C X P}(\mathrm{V})$ \\
\cline { 1 - 1 } 126 & & 7 \\
126 & & 9 \\
86 & & 43 \\
81 & & 15 \\
111 & & 15 \\
111 & & 9 \\
101 & & 5 \\
101 & 13 \\
\hline
\end{tabular}


$<0.30=$ insignificant; between 0.30 and $0.50=$ poor; between 0.50 and $0.70=$ moderate; between 0.70 and $0.90=$ good and $>0.90=$ excellent. For SCC the following criteria were used for analysis (26): $<0.50=$ poor; between 0.50 and $0.75=$ moderate; between 0.75 and $0.90=$ good and $>0.90=$ excellent. For all analyses except $\mathrm{PB}$ and BA the Statistical Package for the Social Sciences (SPSS Inc., EUA) 20.0 version software was used, and for the PB and BA analyses MedCalc (2018 MedCalc Software bvba, Ostend, Belgium) 18.0 version (27) software was used. For all correlations, $P<0.05$ values were considered statistically significant.

\section{Results}

There was no difference between the values of total testosterone, DHT, DHEA and androstenedione before and after the rhCG test when comparing the groups of independent diagnosis at each time of the study (KruskalWallis test: IA total testosterone before $P=0.846$; IA total testosterone after $P=0.726$; total testosterone LC-MS/MS before $P=0.868$; total testosterone LC-MS/MS after $P=0.452$; DHT IA before $P=0.091$; DHT IA after $P=0.284$; DHT LC-MS/MS before $P=0.993$; DHT LC-MS/MS after $P=0.259$; DHEA IA before $P=0.322$; DHEA IA after $P=0.214 ;$ DHEA LC-MS/MS before $P=0.486$; DHEA LC-MS/MS after $P=0.763$; androstenedione IA before $P=0.145$; androstenedione IA after $P=0.595$; androstenedione LC-MS/MS before $P=0.368$; androstenedione LC-MS/MS after $P=0.321)$. For this reason, PAIS, 5AR2D and idiopathic cases were analyzed as a single group (Table 2).

Except for DHEA (Wilcoxon test: $P=0.107$ ), IA values for total testosterone, DHT, DHEA and androstenedione were significantly higher after rhCG test when compared to basal values (Wilcoxon test: total testosterone IA $P<0.001$; total testosterone LC-MS/MS $P<0.001$; DHT IA $P<0.001$; DHT LC-MS/MS $P<0.001$; DHEA LC-MS/MS $P<0.001$; androstenedione IA $P=0.033$; androstenedione LC-MS/MS $P=0.007$ ) (Table 2).

Table 3 shows the correlations between IA and LC-MS/MS. Total testosterone showed an excellent correlation in both ICC and SCC tests, while DHT resulted in a nonexistent and moderate correlation between the two methods in ICC and SCC, respectively; DHEA showed a moderate correlation in both tests and androstenedione with poor and moderate correlation in ICC and SCC, respectively. For total testosterone, the $\mathrm{PB}$ regression did not exclude linear relation $(P=0.09)$

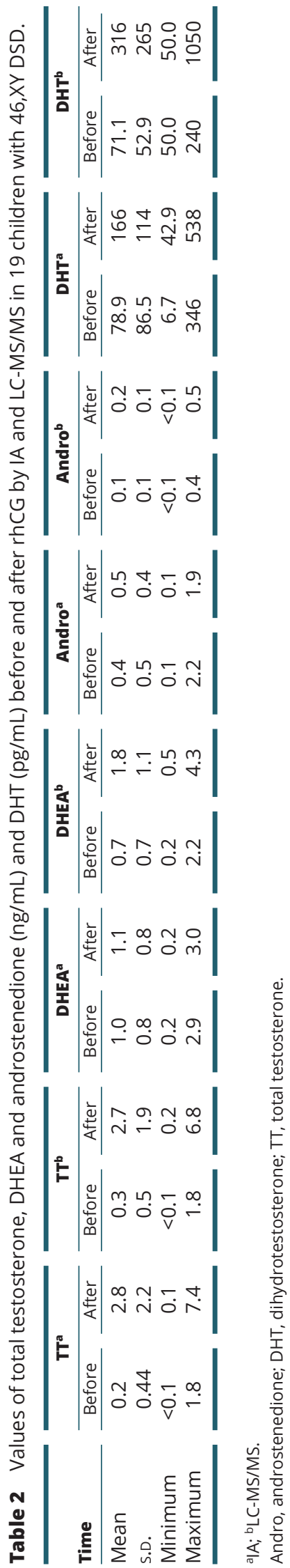


Table 3 Intraclass correlation coefficient (ICC) and Spearman's correlation coefficient (SCC) between the values of total testosterone, DHEA, androstenedione and DHT through IA vS LC-MS/MS of 19 children with 46,XY DSD.

\begin{tabular}{|c|c|}
\hline Total testosterone & DHEA \\
\hline 0.960 (95\% Cl: 0.925-0.979) & 0.585 (95\% Cl: $0.331-0.760)$ \\
\hline$r=0.964 ; P<0.001$ & $r=0.716 ; P<0.001$ \\
\hline
\end{tabular}

$r$, correlation coefficient; $P$, significance level.

and there was no proportional concordance error between the two methods (slope: $1.0540 ; 95 \%$ CI: 0.9641 at 1.1098 ) but there was a systematic error of agreement (intercept: -0.0227 ; 95\% CI: -0.0626 at -0.0182) (Fig. 1A). Though BA graphic showed a mean difference between methods of only 0.05 units (95\% CI: -1.12 at $1.02 \mathrm{ng} / \mathrm{mL}$ ), it was observed a trend for the IA to overestimate total testosterone values in comparison to LC-MS/MS when the mean values of the two methods
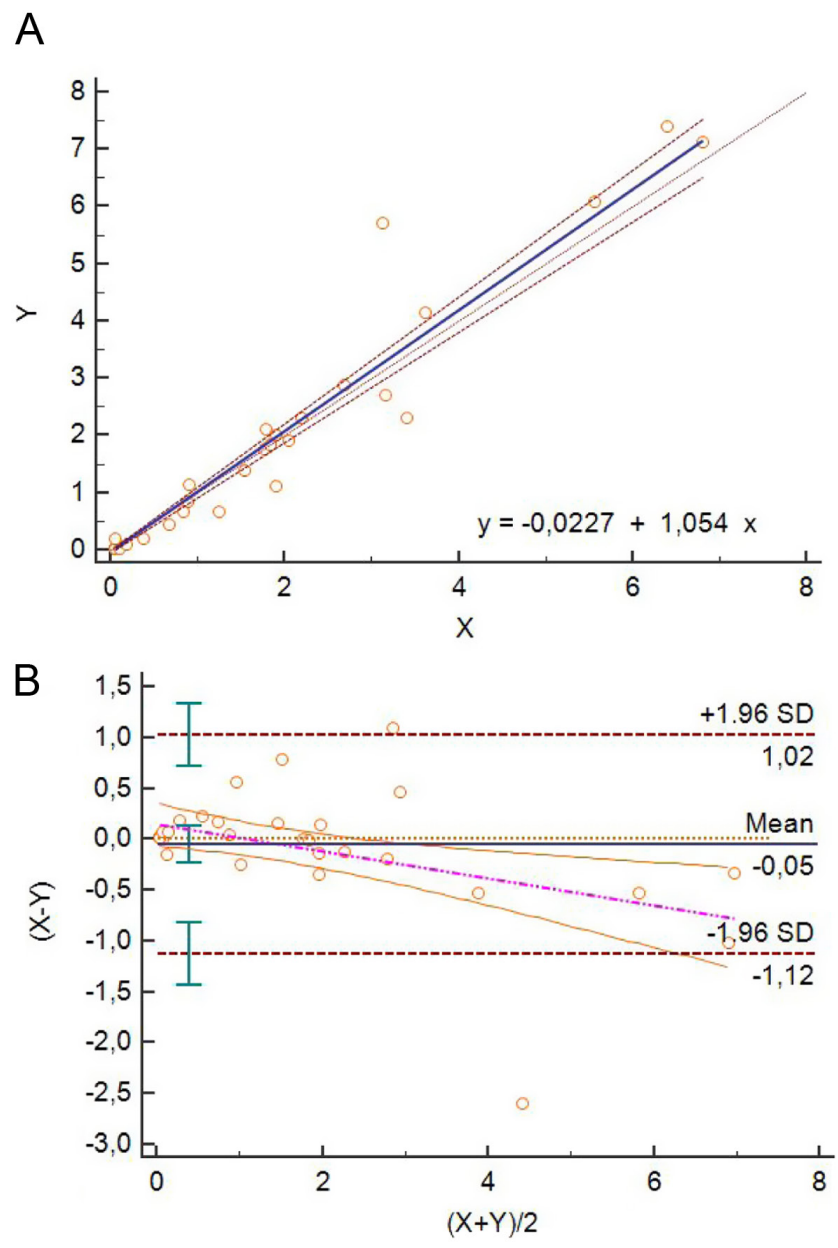

Figure 1

Total testosterone $(\mathrm{ng} / \mathrm{mL})(n=38$ patients with DSD). (A) Passing and Bablok regression for the concordance analysis between IA and LC-MS/MS. (B) Bland and Altman graphic for the concordance analysis between IA and LC-MS/MS. $x=$ LC-MS/MS; $y=I A$.

\begin{tabular}{|c|c|}
\hline Androstenedione & DHT \\
\hline $\begin{array}{c}0.363 \text { (95\% Cl: } 0.053-0.609) \\
r=0.735 ; P<0.001\end{array}$ & $\begin{array}{c}0.222 \text { (95\% Cl: }-1.160 \text { to } 0.514) \\
r=0.631 ; P<0.001\end{array}$ \\
\hline
\end{tabular}

was increased, causing non-constant variation at all concentrations (Fig. 1B).

For DHT, the PB regression did not exclude the linear relation $(P=0.10)$ and did not show proportional (slope: 0.4729; $95 \%$ CI: 0.3650 at 1.6675$)$ or systematic errors (intercept: 12.7857 ; $95 \%$ CI -63.3750 at 29.3500) of concordance (Fig. 2A), despite a great amplitude between the standard deviation of the mean of differences of the two methods and the large CI of each reference line

A

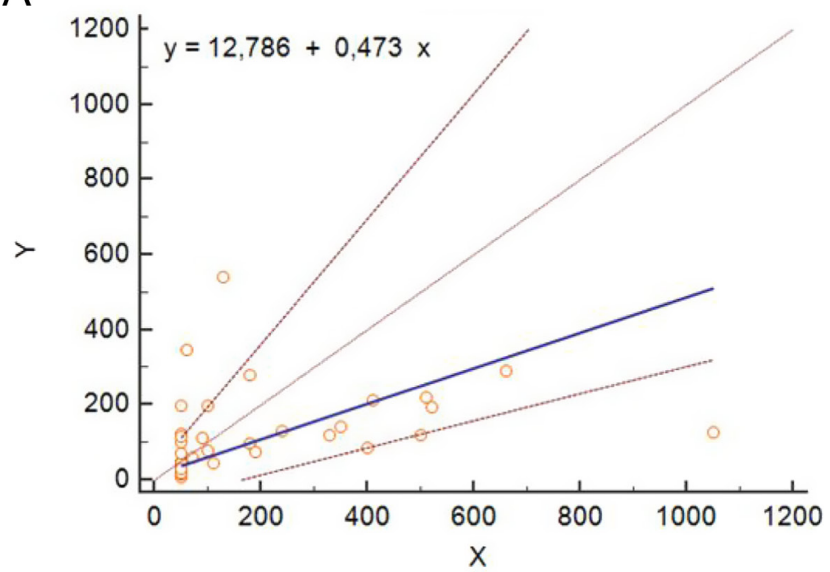

B

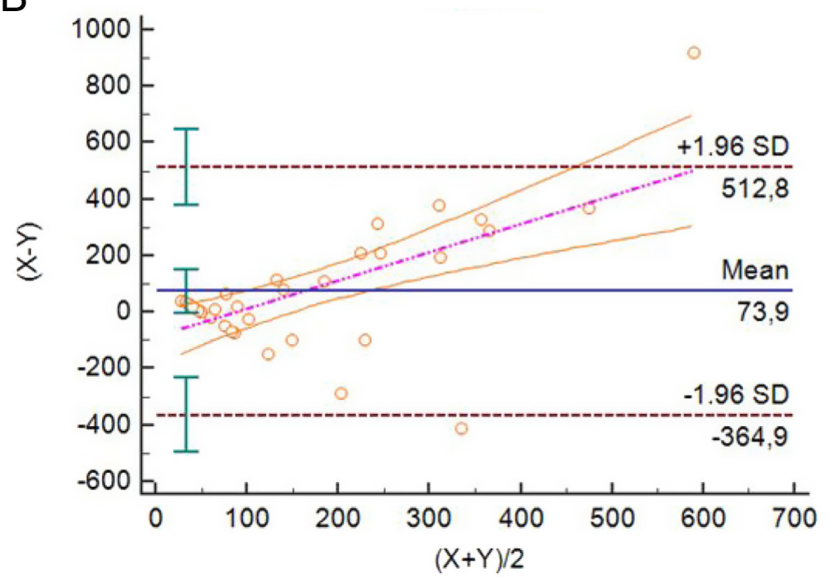

Figure 2

Dihydrotestosterone ( $\mathrm{pg} / \mathrm{mL})(n=35$ patients with DSD). (A) Passing and Bablok for the concordance analysis between IA and LC-MS/MS. (B) Bland and Altman graphic for the concordance analysis between IA and LC-MS/MS. $x=$ LC-MS/MS; $y=I A$.

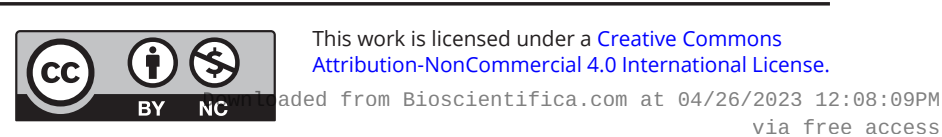


A

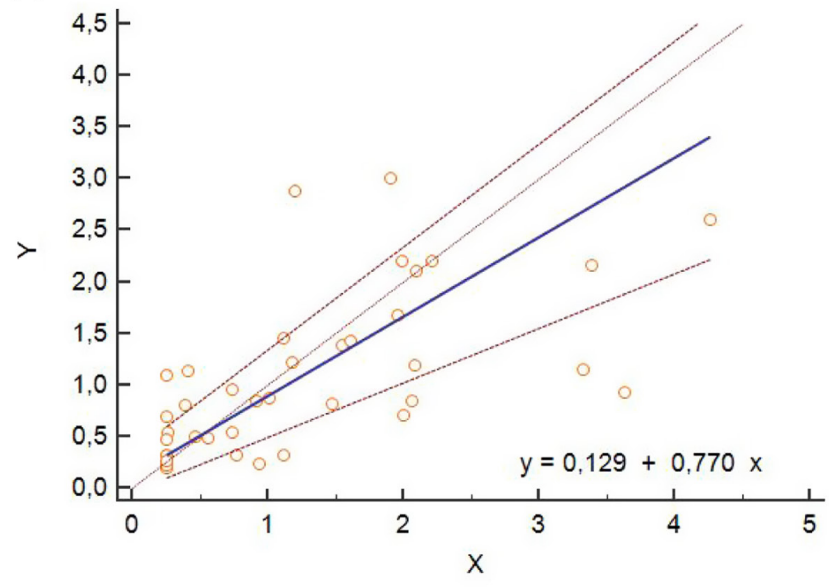

$\mathrm{B}$

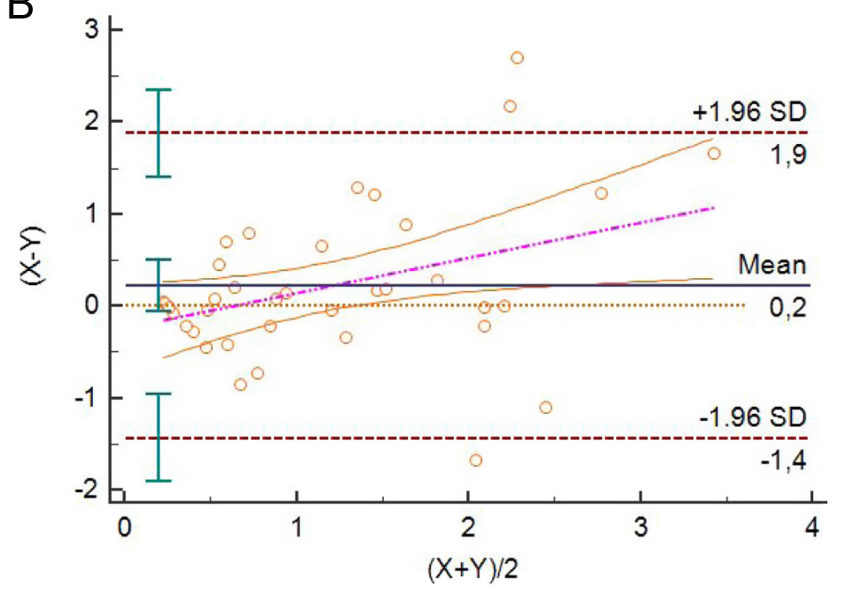

Figure 3

Dehydroepiandrosterone ( $\mathrm{ng} / \mathrm{mL}$ ) ( $n=38$ patients with DSD). (A) Passing and Bablok for the concordance analysis between IA and LC-MS/MS. (B) Bland and Altman graphic for the concordance analysis between IA and LC-MS/MS. $x=$ LC-MS/MS; $y=I A$.

shown in the graphic of Fig. 2B. In the BA graphic, it can be observed an important mean difference of 73.9 units and a trend to high values measured by LC-MS/MS in relation to values measured by IA when the mean of methods was increased (Fig. 2B).

For DHEA, the PB regression did not exclude the linear relation $(P=0.50)$ and it did not present proportional (slope: $0.7704 ; 95 \%$ CI: 0.5287 at 1.0000 ) or systematic error (intercept: 0.1293 ; 95\% CI: -0.0400 at 0.3427 ) of concordance (Fig. 3A). Although the BA graphic shows a mean difference between the methods of only 0.2 $\mathrm{ng} / \mathrm{mL}(95 \% \mathrm{CI}:-1.4$ at $1.9 \mathrm{ng} / \mathrm{mL})$, there was a trend to IA underestimate DHEA values in comparison to the LC-MS/MS values when the mean of the two methods
A

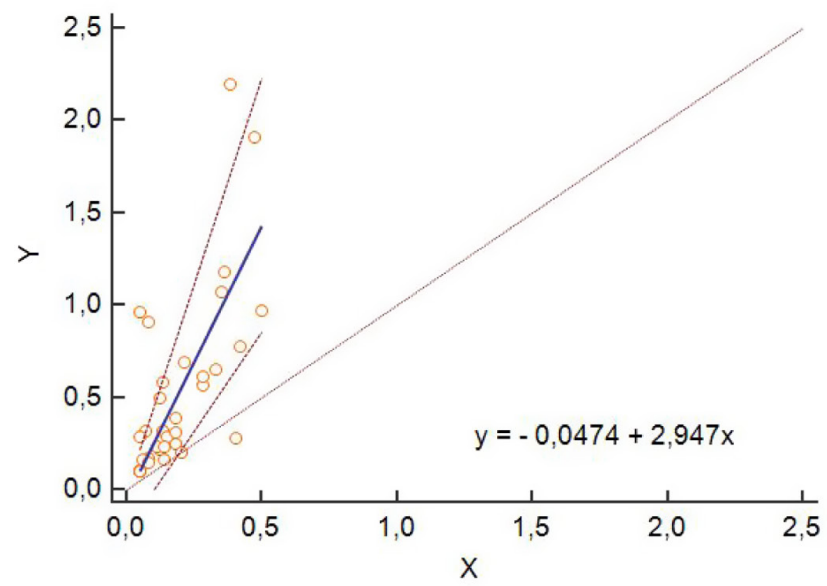

B

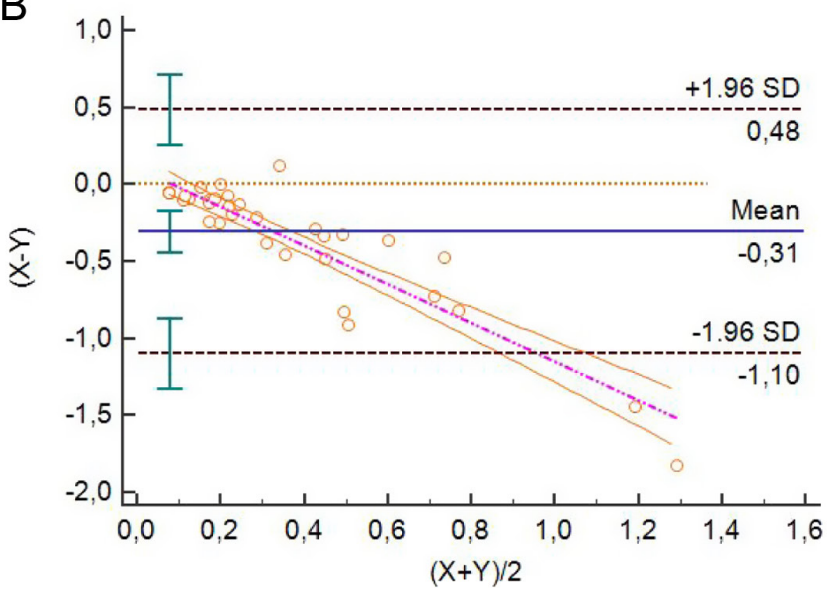

Figure 4

Androstenedione (ng/mL) ( $n=38$ patients with DSD). (A) Passing and Bablok for the concordance analysis between IA and LC-MS/MS. (B) Bland and Altman for concordance analysis between IA and LC-MS/MS.

$\mathrm{x}=\mathrm{LC}-\mathrm{MS} / \mathrm{MS} ; \mathrm{y}=\mathrm{IA}$

was increased, causing non-constant variation at all concentrations (Fig. 3B).

For androstenedione, the $\mathrm{PB}$ regression did not exclude the linear relation $(P=0.07)$, but it showed proportional (slope: $2.9470 ; 95 \%$ CI: 2.1667 at 4.4706 ) and systematic errors of concordance (intercept: -0.04735; 95\% CI: -0.2282 at -0.0083 ) (Fig. 4A). Although the BA graphic shows a mean difference between the methods of only -0.31 units ( $95 \% \mathrm{CI}:-1.10$ at $0.48 \mathrm{ng} / \mathrm{mL}$ ), it was observed a strong trend of IA to overestimate androstenedione values in comparison to LC-MS/MS when the mean of the two methods was increased, causing non-constant variation at all concentrations (Fig. 4B). 


\section{Discussion}

When a laboratory needs to change a method, or install new or alternative methods, it is necessary to use a tool to measure and evaluate the differences between them (28). The validation of a clinical measurement must include all procedures that demonstrate a specific method to be adopted for quantitative evaluation of a variable that has to be trustful and replicable for the intended use $(28,29)$. While correlation tests quantify if two or more variables are correlated, concordance tests evaluate if differences between them are significant $(23,24,28)$. However, a good correlation between the two assay methods does not mean a good concordance between them $(23,24,28)$.

This is the first study comparing IA and LC-MS/MS performances in a specific clinical setting such as prepubertal children with 46,XY DSD after stimulation test with rhCG using androgen measurements usually assessed in those patients. Despite the presence of positive correlation and linearity between the two methods analyzed, they cannot be considered equivalent, as IA is affected by proportional (androstenedione) and systematic (total testosterone and androstenedione) concordance errors, tending to overestimate total testosterone and androstenedione values, and underestimate DHEA and DHT values when compared to LC-MS/MS. Regarding DHT values, we observed the absence of correlation between the measurements of the two evaluated methods and therefore the analysis of agreement is compromised.

This was also the first study to evaluate Leydig cell response to rhCG in prepubertal children diagnosed with $46, \mathrm{XY}$ DSD with known normal testosterone production based on the protocol described by Oliveira et al. (17) because it was the only study that demonstrated the use of hCG as an acute stimulus for gonadal steroidogenesis in its recombinant form, that is potentially free of impurities and contaminations compared to that extracted from the urine of pregnant women with similar properties $(18,28,29)$.

Changes in the estimation of IA results show that this technique may be inappropriate for other clinical situations, especially when small variations in the steroid concentration need to be assessed. Such data suggest that the LC-MS/MS should be conducted for both research and clinical laboratories, enabling the detection of subtle alterations needed for either the diagnosis or the follow-up treatment in specific clinical disorders, such as DSD (30, 31,32 ). Another common pitfall associated with IA is cross-reactivity between structurally similar molecules and the antibody used for detection. Cross-reactivity is of particular concern when those similar molecules are present in much higher abundance than the antigen of interest, as is the case with testosterone in relation to DHT (33).

Roli et al. (19), evaluating the rhCG response to gonadal and adrenal androgens in 13 adult patients with Klinefelter syndrome, also observed good linearity between IA and LC-MS/MS and a trend to overestimate IA in relation to LC-MS/MS values, similar to the results presented in this study.

The most important issue remains the availability of the reference intervals calibrated in relation to the trial $(31,34)$. Several clinical diagnoses depend on the specific limitation of the steroids of interest. For example, according to various studies carried out in prepubertal children with 46,XY DSD, total testosterone after stimulation with uhCG must increase from 1.1 to $1.5 \mathrm{ng} / \mathrm{mL}$ $(15,21)$, may or may not indicate normal testosterone secretion by Leydig cells on this patients, and, therefore, determine which molecular investigation must be done. In the case of inappropriate testosterone secretion in children with $46, \mathrm{XY}$ DSD, the investigation must be directed to hypogonadotropic hypogonadism, gonadal dysgenesis, Leydig cell hypoplasia (LHCGR defect) or defects in testosterone biosynthesis, against the normal or increased testosterone which directs to the molecular investigation of PAIS/CAIS or 5AR2D $(11,12,35,36)$.

The results of the present study suggest that the difference between IA and LC-MS/MS must be carefully assessed in treatments for which androgen levels are important to determine the pharmacologic manipulation, such as the management of children with congenital adrenal hyperplasia. This methodological limit is even more important in clinical conditions characterized by low androgen levels. It is valid to say that the results found in this study are expected for other populations without the diagnosis of DSD.

It is worth emphasizing that the average of DHT values found in the present study by LC-MS/MS after stimulation was much higher than the one by IA (316.67 and $166.82 \mathrm{pg} / \mathrm{mL}$, respectively), which suggests that the LC-MS/MS method was more specific for DHT dosage. Probably, conventional IA techniques are not able to detect all the concentration present in the sample due to the low sensitivity of the antibody used, considering that the serum concentration of DHT is much lower than other steroids. We demonstrated that the comparison is worse in post-stimulation samples by ICC (0.053 in post-stimulation and 0.235 in pre-stimulation samples). Probably other teriodal compounds are present at 
unconventionally high levels interfering with IA measure of DHT or the dynamic range is not wide enough to measure DHT.

Despite the small number of cases analyzed, which is justified when dealing with rare disorders, about $76 \%$ (19 of 25 cases) of the patients available in the service accepted to participate in the study. The strong points of the study are the accuracy of the etiological diagnosis of cases included and the statistical analysis applied.

In the last few years, many efforts have been done to improve the two methods. If on one side the new IA generation is being validated taking into consideration the comparison with MS, on the other side, LC-MS/MS is rapidly enabling its facilitated use and offering a wide range of applications. In a short future, MS may not replace, but rather, complement IA in both research and clinical practice. Decisions about diagnosis and treatment are currently taken considering the cut-points proposed by IA, whereas that use of LC-MS/MS in clinical laboratories must be preceded by a diagnose threshold revision and CIs. The clinical practice and research must contribute to improve the sustainability and accuracy of IA and LC-MS/MS.

In conclusion, though LC-MS/MS and IA methods presented good linearity and some correlation between them, systematic and/or proportional concordance errors were detected, besides an evident tendency of higher values measured by IA. Therefore, the results of androgens evaluated by IA must be carefully analyzed.

\section{Declaration of interest}

The authors declare that there is no conflict of interest that could be perceived as prejudicing the impartiality of the research reported.

\section{Funding}

CNPq (Process: 472098/2011-0), FAEPEX (Process: 106/2014) and CAPES (Process: 0001)

\section{Author contribution statement}

L R O designed the study, performed experiments and co-wrote the paper. $C A L$ and $A T M G$ designed the study and co-wrote the paper. G G F, J L C, $R L, D A S, M I C$ and M P M performed experiments and co-wrote the paper. A M M performed statistical analyses. G G J designed the study, performed experiments, supervised the research and co-wrote the paper.

\section{Acknowledgements}

To Merck for the donation of rhCG samples and to the Fleury Group for the collaboration with the DHT dosages by LC-MS/MS.

\section{References}

1 Honour JW. Steroid assays in paediatric endocrinology. Journal of Clinical Research in Pediatric Endocrinology 20102 1-16. (https://doi. org/10.4274/jcrpe.v2i1.1)

2 Dekkers OM, Horváth-Puhó E, Cannegieter SC, Vandenbroucke JP, Sørensen HT \& Jørgensen JO. Acute cardiovascular events and all-cause mortality in patients with hyperthyroidism: a populationbased cohort study. European Journal of Endocrinology 2017176 1-9. (https://doi.org/10.1530/EJE-16-0576)

3 Audi L, Camats N, Fernández-Cancio M \& Granada ML. Development of laboratory investigations in disorders of sex development. Sexual Development 201812 7-18. (https://doi. org/10.1159/000479719)

4 Taylor AE, Keevil B \& Huhtaniemi IT. Mass spectrometry and immunoassay: how to measure steroid hormones today and tomorrow. European Journal of Endocrinology 2015173 D1-D12. (https://doi.org/10.1530/EJE-15-0338)

5 Albrecht L \& Styne D. Laboratory testing of steroid gonadal in children. Pediatric Endocrinology Reviews 20075 (Supplement 1) 599-607.

6 Handelsman DJ \& Wartofsky L. Requirement for mass spectrometry sex steroid assays in the Journal of Clinical Endocrinology and Metabolism. Journal of Clinical Endocrinology and Metabolism 201398 3971-3973. (https://doi.org/10.1210/jc.2013-3375)

7 Krone N, Hughes BA, Lavery GG, Stewart PM, Arlt W \& Shackleton CHL. Gas chromatography/mass spectrometry (GC) MS) remains a pre-eminent discovery tool in clinical steroid investigations even in the era of fast liquid chromatography tandem mass spectrometry (LC/MS/MS). Journal of Steroid Biochemistry and Molecular Biology 2010121 496-504. (https://doi.org/10.1016/j. jsbmb.2010.04.010)

8 Stanczyk FZ \& Clarke NJ. Advantages and challenges of mass spectrometry assays for steroid hormones. Journal of Steroid Biochemistry and Molecular Biology 2010121 491-495. (https://doi. org/10.1016/j.jsbmb.2010.05.001)

9 Cao ZT, Botelho JC, Rej R \& Vesper H. Accuracy-based proficiency testing for testosterone measurements with immunoassays and liquid chromatography mass spectrometry. Clinica Chimica Acta: International Journal of Clinical Chemistry 2017469 31-36. (https:// doi.org/10.1016/j.cca.2017.03.010)

10 Rosner W, Auchus RJ, Azziz R, Sluss PM \& Raff H. Position statement: utility, limitations, and pitfalls in measuring testosterone: an Endocrine Society position statement. Journal of Clinical Endocrinology and Metabolism 200792 405-413. (https://doi.org/10.1210/jc.20061864)

11 Lee PA, Houk CP, Ahmed SF, Hughes IA \& International Consensus Conference on Intersex organized by the Lawson Wilkins Pediatric Endocrine Society and the European Society for Paediatric Endocrinology. Consensus statement on management of intersex disorders. International Consensus Conference on Intersex. Pediatrics 2006118 e488-e500. (https://doi.org/10.1542/peds.2006-0738)

12 Lee PA, Nordenström A, Houk CP, Ahmed SF, Auchus R, Baratz A, Dalke KB, Liao LM, Lin-Su K, Looijenga LHJ, et al. Global disorders of sex development update since 2006: perceptions, approach and care. Hormone Research in Paediatrics 201685 158-180. (https://doi. org/10.1159/000442975)

13 Cox K, Bryce J, Jiang J, Rodie M, Sinnott R, Alkhawari M, Arlt W, Audi L, Balsamo A, Bertelloni S, et al. Novel associations in disorders of sex development: findings from the I-DSD Registry. Journal of Clinical Endocrinology and Metabolism 201499 E348-E355. (https:// doi.org/10.1210/jc.2013-2918)

14 De Paula GB, Barros BA, Carpini S, Tincani BJ, Mazzola TN, Sanches Guaragna M, Piveta CS, de Oliveira LC, Andrade JG, GuaragnaFilho G, et al. 408 Cases of genital ambiguity followed by single multidisciplinary team during 23 years: etiologic diagnosis and sex

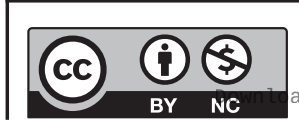

This work is licensed under a Creative Commons Attribution-NonCommercial 4.0 International License. ded from Bioscientifica.com at 04/26/2023 12:08:09PM 
of rearing. International Journal of Endocrinology 201620164963574. (https://doi.org/10.1155/2016/4963574)

15 Bertelloni S, Russo G \& Baroncelli GI. Human chorionic gonadotropin test: old uncertainties, new perspectives, and value in 46,XY disorders of sex development. Sexual Development 201812 41-49. (https://doi.org/10.1159/000481552)

16 Ahmed SF, Keir L, McNeilly J, Galloway P, O’Toole S \& Wallace AM. The concordance between serum anti-Mullerian hormone and testosterone concentrations depends on duration of hCG stimulation in boys undergoing investigation of gonadal function. Clinical Endocrinology 201072 814-819. (https://doi.org/10.1111/j.13652265.2009.03724.x)

17 Oliveira LR, Homma TK, Woloszynek RR, Brito VN \& Longui CA. Gonadal response after a single-dose stimulation test with recombinant human chorionic gonadotropin (r-hCG) in patients with isolated prepubertal cryptorchidism. Basic and Clinical Andrology 201626 13. (https://doi.org/10.1186/s12610-016-0039-2)

18 Cailleux-Bounacer A, Reznik Y, Cauliez B, Menard JF, Duparc C \& Kuhn JM. Evaluation of endocrine testing of Leydig cell function using extractive and recombinant human chorionic gonadotropin and different doses of recombinant human LH in normal men. European Journal of Endocrinology 2008159 171-178. (https://doi org/10.1530/EJE-07-0876)

19 Roli L, Santi D, Belli S, Tagliavini S, Cavalieri S, De Santis MC, Baraldi E, Fanelli F, Mezzullo M, Granata AR, et al. The steroid response to human chorionic gonadotropin (hCG) stimulation in men with Klinefelter syndrome does not change using immunoassay or mass spectrometry. Journal of Endocrinological Investigation $2017 \mathbf{4 0}$ 841-850. (https://doi.org/10.1007/s40618-017-0653-7)

20 Tomlinson C, Wallace AM \& Ahmed SF. Erroneous testosterone assay causing diagnostic confusion in a newborn infant with intersex anomalies. Acta Paediatrica 200493 1004-1005. (https://doi. org/10.1111/j.1651-2227.2004.tb02704.x)

21 Stuchi-Perez EG, Hackel C, Oliveira LE, Ferraz LF, Oliveira LC, NunesSilva D, Toralles MB, Steinmetz L, Damiani D, Maciel-Guerra AT, et al. Diagnosis of 5alpha-reductase type 2 deficiency: contribution of anti-Müllerian hormone evaluation. Journal of Pediatric Endocrinology and Metabolism 200518 1383-1389. (https://doi.org/10.1515/ jpem.2005.18.12.1383)

22 Scientific Working Group for Forensic Toxicology (SWGTOX). Standard practices for method validation in forensic toxicology. Report from the Scientific Working Group for Forensic Toxicology. Journal of Analytical Toxicology 201337 452-474. (https://doi. org/10.1093/jat/bkt054)

23 Passing $\mathrm{H} \&$ Bablok W. A new biometrical procedure for testing the equality of measurements from two different analytical methods. Application of linear regression procedures for method comparison studies in clinical chemistry, part I. Journal of Clinical Chemistry and Clinical Biochemistry 198321 709-720. (https://doi.org/10.1515/ cclm.1983.21.11.709)
24 Bland JM \& Altman DG. Measuring agreement in method comparison studies. Statistical Methods in Medical Research 19998 135-160. (https://doi.org/10.1177/096228029900800204)

25 Mukaka MM. Statistics corner: a guide to appropriate use of correlation coefficient in medical research. Malawi Medical Journal 201224 69-71.

26 Koo TK \& Li MY. A guideline of selecting and reporting intraclass correlation coefficients for reliability research. Journal of Chiropractic Medicine 201615 155-163. (https://doi.org/10.1016/j. jcm.2016.02.012)

27. MedCalc.MedCalc Statistical Software version 18. Ostend, Belgium: MedCalc Software bvba, 2018. (available at: http://www.medcalc.org)

28 Giavarina D. Understanding Bland Altman analysis. Biochemia Medica 201525 141-151. (https://doi.org/10.11613/BM.2015.015)

29 Hirakata VN \& Camey AS. Análise de concordância entre os métodos de Bland-Altman. Revista do Hospital de Clinicas de Porto Alegre 2009 29 261-268.

30 Courant F, Aksglaede L, Antignac JP, Monteau F, Sorensen K, Andersson AM, Skakkebaek NE, Juul A \& Le Bizec BL. Assessment of circulating sex steroid levels in prepubertal and pubertal boys and girls by a novel ultrasensitive gas chromatography-tandem mass spectrometry method. Journal of Clinical Endocrinology and Metabolism 201095 82-92. (https://doi.org/10.1210/jc.2009-1140)

31 Buttler RM, Martens F, Fanelli F, Pham HT, Kushnir MM, Janssen MJW, Owen L, Taylor AE, Soeborg T, Blankenstein MA, et al. Comparison of 7 published LC-MS/MS methods for the simultaneous measurement of testosterone, androstenedione, and dehydroepiandrosterone in serum. Clinical Chemistry 201561 1475-1483. (https://doi.org/10.1373/clinchem.2015.242859)

32 Ahmed SF, Achermannt JC, Arlt W, Balen AH, Conway G, Edwards ZL, Elford S, Hughes IA, Izatt L, Krone N, et al. UK guidance on the initial evaluation of an infant or an adolescent with a suspected disorder of sex development. Clinical Endocrinology 201175 12-26. (https://doi.org/10.1111/j.13652265.2011.04076.x)

33 Yarrow JF, Beck DT, Conover CF, Beggs LA, Goldberger BA \& Borst SE. Invalidation of a commercially available human 5a-dihydrotestosterone immunoassay. Steroids 201378 1220-1225. (https://doi.org/10.1016/j.steroids.2013.08.013)

34 Pitt JJ. Principles and applications of liquid chromatography mass spectrometry in clinical biochemistry. Clinical Biochemistry Reviews 200930 19-34.

35 Mendonça BB, Batista RL, Domenice S, Costa EM, Arnhold IJ, Russell DW \& Wilson JD. Steroid 5 $\alpha$-reductase 2 deficiency. Journal of Steroid Biochemistry and Molecular Biology 2016163 206-211.

36 de Andrade Machado Neto F, Moreno Morcillo A, Trevas Maciel-Guerra A \& Guerra-Junior G. Idiopathic male pseudohermaphroditism is associated with prenatal growth retardation. European Journal of Pediatrics 2005164 287-291. (https:// doi.org/10.1007/s00431-005-1626-y)

Received in final form 15 September 2020

Accepted 7 October 2020

Accepted Manuscript published online 8 October 2020 https://ec.bioscientifica.com https://doi.org/10.1530/EC-20-0454 (c) 2020 The authors Published by Bioscientifica Ltd

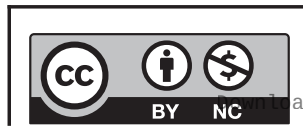

This work is licensed under a Creative Commons Attribution-NonCommercial 4.0 International License. ded from Bioscientifica.com at 04/26/2023 12:08:09PM 